“(C) 2018 IEEE. Personal use of this material is permitted. Permission from IEEE must be obtained for all other uses, in any current or future media, including reprinting/republishing this material for advertising or promotional purposes, creating new collective works, for resale or redistribution to servers or lists, or reuse of any copyrighted component of this work in other works." 


\title{
A Novel Optimal Sliding Mode Control For Multiple Time-Delay Systems
}

\author{
Ahmadreza Argha ${ }^{1}$, Steven W. Su${ }^{2}$, Andrey Savkin ${ }^{1}$, and Branko G. Celler ${ }^{1}$
}

\begin{abstract}
This paper considers the problem of delayindependent optimal sliding mode control design for uncertain systems with multiple constant delays. An improved delayindependent framework for the design of SMC is established in terms of a linear matrix inequality for time-delay systems, in which multi-channel $\mathscr{H}_{2}$ performances of the closed-loop system are under control. Unlike most of the existing methods, the required level of control effort to maintain sliding will be taken into account in this new framework. Our two-stage SMC is constructed as follows. Firstly, a certain state feedback gain is designed while assigning some of the closed-loop eigenvalues precisely to a predetermined stable location as well as ensuring a prescribed multi-channel $\mathscr{H}_{2}$ performance level of the closedloop system. In the second stage, we will find the optimal switching surface associated with the gain designed in the first stage via a novel approach developed for this goal while ensuring the stability of the reduced-order dynamics.
\end{abstract}

\section{INTRODUCTION}

Dealing with time-delay systems is crucial in practical control systems. Broadly speaking, the necessity of considering time delays in the systems to be controlled is twofold. Firstly, time delays are employed to model systems more accurately. Indeed, a large number of practical systems including manufacturing processes involve this phenomena in their dynamics owing to the deployment of sensors, actuators and communication networks. Next, time delays are helpful as a modeling tool to simplify some infinite-dimensional systems [1]. Different control schemes have been proposed for time-delay systems such as $\mathscr{H}_{\infty}$ control [2].

Mostly, conventional SMC design methods, including the approaches proposed for time delay systems, are unable to take into account the control action required for inducing and maintaining sliding. The reason can be found in the two-stage nature of the conventional SMC design schemes employed in the existing literature. Indeed, in the most of the current SMC design schemes, while the switching function is synthesized in the first stage, the control action required to induce and maintain sliding is not possible to be penalized [3]. Clearly, in such cases, a impractical switching surface and thereby a control law may be selected.

A few investigators, in the related field, have considered this problem, e.g. [3], [4], [5]. In this different thread of SMC design, which can be referred to as LQR-based SMC design schemes, the major idea is to select the switching surface

\footnotetext{
${ }^{1}$ School of Electrical Engineering and Telecommunications, University of New South Wales, Sydney, NSW 2052, Australia.

$\{a \cdot$ argha, a.savkin, b.celler\}@unsw.edu.au

2 Faculty of Engineering and Information Technology, University of Technology, Sydney, PO Box 123, Broadway, NSW 2007, Australia. Steven.Su@uts.edu.au
}

while minimizing an index function of the system state and control input. However, not necessarily any arbitrary weighting matrices in the index function can lead to a sliding mode control. To solve this problem, in [4], as the main focus is on the single-input systems, it is ensured that at least one of the closed-loop system eigenvalues is a real value. Hence, the authors of [4] propose to either reselect the weighting matrices or approximate the closed-loop system eigenvalues so that a new set of eigenvalues are derived which is dividable into the null-space and range-space dynamics. To resolve two main drawbacks of [4], ie, 1) loss of optimality and/or robustness due to the random approximation of eigenvalues, 2) not giving a rigorous method on how to reselect the weighting matrices, of the approach in [4], the paper [5] has proposed to search for a weighting matrix which is the closest to the desired one and more importantly generates the desired closed-loop eigenvalues. It is worthwhile noting that the both methods in [4], [5] are only appropriate for single input systems. Additionally, in order to design an optimal sliding mode control that is able to penalize the control effort required to maintain sliding, two new methods are introduced in [3]. Both of these methods are constructed in a specific coordinate system achieved by using two preliminary coordinate transformations. Finally, it should be noted that none of the above cited-papers, considering the problem of optimal SMC design, considers time delay systems and the proposed schemes may not simply be extended to time delay systems.

This paper aims at developing a novel optimal SMC for the systems involving time delay systems and proposes a novel method for this purpose that offers several advantages to the field. This approach employs a specific partial eigenstructure assignment method to assign $m$ (ie, the number of system inputs) arbitrary stable real eigenvalues while an appropriate sliding motion dynamics will be guaranteed by enforcing different Lyapunov-type constraints such as the multi-channel $\mathscr{H}_{2}$ constraints.

Notation: $\operatorname{herm}(E)$, where $E$ is a square matrix, stands for $E+E^{*}$.

\section{Problem Statement And Preliminaries}

Consider the following linear-time-invariant (LTI) system with multiple constant time-delays:

$$
\begin{aligned}
\dot{x}(t) & =\sum_{i=0}^{h} A_{i} x\left(t-\tau_{i}\right)+B_{2}[u(t)+f(x, u, t)], \\
x(t) & =\phi(t), \quad t \in[-\tau, 0], \quad \tau=\left.\max \left(\tau_{i}\right)\right|_{i=1} ^{h}, \\
z(t) & =C x(t)+D u(t),
\end{aligned}
$$


where $x \in \mathbb{R}^{n}, u \in \mathbb{R}^{m}$, and $z \in \mathbb{R}^{p}$ are the system state, control input, and $\mathscr{H}_{2}$ performance output signal, respectively. It is further assumed that all the system matrices are constant and have appropriate dimensions. The unknown signal $f(x, u, t): \mathbb{R}^{n} \times \mathbb{R}^{m} \times \mathbb{R}_{+} \rightarrow \mathbb{R}^{m}$ denotes matched uncertainty in (1) whose Euclidean norm is bounded by a known function $\rho(x, u, t)$. We further, without loss of generality, assume that $m \leq p \leq n$, and $\operatorname{rank}\left(B_{2}\right)=m$. Here $\tau_{0}=0$ and $\tau_{i} \geq 0, i=$ $1, \cdots, h$ are constant delays. $\phi(t)$ is a continuous vectorvalued initial function defined on $t \in[-\tau, 0]$.

Now we consider a linear switching surface as follows:

$$
\mathscr{S}=\{x: s(t) \triangleq F x(t)=0\},
$$

where $F \in \mathbb{R}^{m \times n}$ is a full rank matrix designed in this paper so that the resulting reduced order sliding mode, when the system states are steered to $\mathscr{S}$, has suitable dynamics. Now consider the following control law:

$$
u(t)=-\left(F B_{2}\right)^{-1}\left(F A_{0}-\Delta F\right) x(t)+\xi(t),
$$

where $\Delta \in \mathbb{R}^{m \times m}$ is a prescribed stable matrix, and $\xi(t) \in$ $\mathbb{R}^{m}$ denotes the discontinuous component in the sliding mode controller. Now the nonlinear part of the controller is proposed as:

$$
\xi(t)=-\left(F B_{2}\right)^{-1}\left[\varphi+\left\|F B_{2}\right\| \rho(x, u, t)\right] \frac{s(t)}{\|s(t)\|} \quad \text { if } s(t) \neq 0
$$

in which $\varphi>0$ is a scalar gain to be designed in the sequel of the paper. It is also assumed that $\Delta=\delta I_{m}$, where $\delta$ is a given negative scalar. As $\Delta=\delta I_{m}$ can commute with $F$, the control law $u(t)$ in (3) can be reformulated as

$$
u(t)=\left(F B_{2}\right)^{-1} F A_{0}^{\delta} x(t)+\xi(t),
$$

where $A_{0}^{\delta}=\delta I_{n}-A_{0}$. Now let $f(\cdot)=0$ in (1). We then assume the controller in (5) contains only the linear part; ie, $\xi(\cdot)=0$, therefore

$$
\begin{aligned}
\dot{x}(t) & =\sum_{i=0}^{h} A_{i} x\left(t-\tau_{i}\right)+B_{2} u(t)+B_{1} w(t) \\
z(t) & =C x(t)+D u(t) \\
u(t) & =\left(F B_{2}\right)^{-1} F A_{0}^{\delta} x(t),
\end{aligned}
$$

where $w(t)$ is an artificial mismatched disturbance and the distribution matrix $B_{1}$ is of appropriate dimension. The objective of this paper is to find a sliding matrix $F$ so that the resulting reduced order motion, when restricted to $\mathscr{S}$, is stable and meets multi-channel $\mathscr{H}_{2}$ performance specifications. For this target, one may resort to solve a multi-channel $\mathscr{H}_{2}$ state feedback problem and thereby find the corresponding switching matrix. This trivial scheme may not necessarily lead to any solution, unless the obtained state feedback gain $K$ can ensure that $m$ of the closed-loop poles are exactly located at $\delta$. In short, for the design of a multichannel $\mathscr{H}_{2}$-based SMC, we need to address the following two problems:

Problem 1: Blend the multi-channel $\mathscr{H}_{2}$ problem with the partial eigenstructure assignment method, ie, design a feedback gain $K$ enforcing the multi-channel $\mathscr{H}_{2}$ constraints while ensuring $m$ poles of the closed-loop system are precisely located at $\delta$.

Problem 2: Select the switching matrix $F$ associated with the particular matrix gain $K$, derived in Problem 1

The above-stated problems are dealt with in the following two sections.

\section{Eigenstructure Assignment Problem For $\mathscr{H}_{2}$-BASED SMC DESIGN}

In the current section, we develop an LMI characterization of the multi-channel $\mathscr{H}_{2}$ problem for the systems involving multiple constant time-delays, which leads to potentially less conservative results compared to the so-called quadratic approach. It will also be very useful for the novel SMC of this paper, as it allows to develop a partial eigenstructure assignment scheme ensuring precise locations in terms of LMIs. Let us assume temporarily that there is no matched uncertainty in (1), ie, $f(x, u, t)=0$.

\section{A. $\mathscr{H}_{2}$ LMI characterization}

Lemma 1: The following statements, involving symmetric matrix variables $X, Z$ and $W_{i}, i=1, \cdots, h$, and the general matrix variables $Y$ and $G$ are equivalent.

i) $\exists K$ such that $\sum_{i=0}^{h} A_{i} e^{-s \tau_{i}}+B_{2} K$ is stable and $\left\|(C+D K)\left[s I-\left(\sum_{i=0}^{h} A_{i} e^{-s \tau_{i}}+B_{2} K\right)\right]^{-1} B_{1}\right\|_{2}^{2}<\gamma$

ii) $\exists X>0, Z>0, Y$, and $W_{i}>0, i=1, \cdots, h$ such that

$$
\begin{aligned}
& {\left[\begin{array}{ccc}
\Phi_{11} & \star & \star \\
\operatorname{vec}(X)_{i=1}^{h} & -\operatorname{diag}\left(W_{i}\right)_{i=1}^{h} & \star \\
C X+D Y & 0 & -\gamma I
\end{array}\right]<0,} \\
& {\left[\begin{array}{cc}
-Z & \star \\
B_{1} & -X
\end{array}\right]<0,} \\
& \operatorname{trace}(Z)<1,
\end{aligned}
$$

where $\Phi_{11}:=A_{0} X+X A_{0}^{T}+B_{2} Y+Y^{T} B_{2}^{T}+\sum_{i=1}^{h} A_{i} W_{i} A_{i}^{T}$ and $Y=K X$.

iii) $\exists X>0, Z>0, W_{i}>0, i=1, \cdots, h, Y$, and $G$ such that

$\left[\begin{array}{cccr}X-\left(G+G^{T}\right) & \star & \star & \star \\ \Phi_{21} & \Phi_{22} & \star & \star \\ \operatorname{vec}(G)_{i=1}^{h} & 0 & -v^{-1} \operatorname{diag}\left(W_{i}\right)_{i=1}^{h} & \star \\ C G+D Y & 0 & 0 & -v^{-1} \gamma I\end{array}\right]<0$,

$\left[\begin{array}{cc}-Z & \star \\ B_{1} & -X\end{array}\right]<0$

$\operatorname{trace}(Z)<1$,

where $\Phi_{21}:=v\left(A_{0} G+B_{2} Y\right)+G, \quad \Phi_{22}:=-X+$ $v \sum_{i=1}^{h} A_{i} W_{i} A_{i}^{T}, 0<v \ll 1$ is a constant scalar, and $Y=K G$.

Proof: The proof is given in the Appendix section.

Remark 1: The controller (5) is a rational controller, see [6]. However, considering a system with constant delays, it is possible to employ a non-rational control strategy here. In this work, the nonlinear part of the sliding mode controller is used in order to cope with the influence of uncertainty arisen from time delays in the system. This feature indeed makes 
this scheme applicable to the systems with time varying delays.

\section{B. Multi-channel $\mathscr{H}_{2}$ state feedback using improved LMI characterizations}

Now let $T_{w z}(s)$ denotes the closed-loop transfer function from $w$ to $z$ for control law $u=K x$. Our target is to compute a matrix $K$ which meets several performance specifications of the form

$$
\begin{aligned}
& \operatorname{minimize}\left\|T_{w_{i} z_{i}}\right\|_{2} \\
& \text { subject to }\left\|T_{w_{1} z_{1}}\right\|_{2}^{2}<\gamma_{1}, \cdots,\left\|T_{w_{i-1} z_{i-1}}\right\|_{2}^{2}<\gamma_{i-1}, \\
&\left\|T_{w_{i+1} z_{i+1}}\right\|_{2}^{2}<\gamma_{i+1}, \cdots,\left\|T_{w_{\mathscr{N}} z_{\mathscr{N}}}\right\|_{2}^{2}<\gamma_{\mathscr{N}},
\end{aligned}
$$

where $\left\|T_{w_{i} z_{i}}\right\|_{2}:=\left\|L_{i} T_{w z} R_{i}\right\|_{2}$, in which $L_{i}$ and $R_{i}$ are used to specify the involving channel in the associated constraint. In the sequel of this paper, we use $\mathscr{N}$ to denote the number of channels or the independent Lyapunov variables. Furthermore, a realization of $T_{w_{i} z_{i}}$ is achieved by replacing $B_{1}, C$ and $D$ by $B_{1, i}, C_{i}$ and $D_{i}, i=1, \cdots, \mathscr{N}$, respectively, in (1). The closed-loop performance can be guaranteed by constraining (minimizing) the $\mathscr{H}_{2}$ performance of the closed-loop systems related to (input/output) signals $w_{i}=R_{i} w$ and $z_{i}=L_{i} z$; see [7], [8]. Suppose that each channel is associated with a set of LMI constraints presented in (7), (8), and (9). Then the LMI characterization for state feedback synthesis with multichannel $\mathscr{H}_{2}$ specifications will be achieved by assigning a different Lyapunov variable $X_{i}>0$ to every channel and using common variables $G$ and $Y$ for all channels. As a result, using the item iii) of Lemma 1, the LMI characterization for the channel $l$ can be represented as:

$$
\left[\begin{array}{cccc}
X_{l}-\left(G+G^{T}\right) & \star & \star & \star \\
\Phi_{21} & \tilde{\Phi}_{22} & \star & \star \\
\operatorname{vec}(G)_{i=1}^{h} & 0 & -v^{-1} \operatorname{diag}\left(W_{l i}\right)_{i=1}^{h} & \star \\
C_{l} G+D_{l} Y & 0 & 0 & -v^{-1} \gamma_{l} I
\end{array}\right]<0,
$$

$\left[\begin{array}{cc}-Z_{l} & \star \\ B_{1, l} & -X_{l}\end{array}\right]<0$,

$\operatorname{trace}\left(Z_{l}\right)<1$,

where $\tilde{\Phi}_{22}:=-X+v \sum_{i=1}^{h} A_{i} W_{l i} A_{i}^{T}, 0<v \ll 1, X_{l}>0, Z_{l}>$ $0, W_{l i}>0, i=1, \cdots, h, G$ and $Y$ are LMI variables, and further, $Y=K G$. Thereby the optimization problem in 10 can be cast as

$$
\text { minimize } \gamma_{i}
$$

subject to (11), (12), and (13) for i-th channel, (11), 12, and (13) for $\mathrm{j}$-th channel with given $\gamma_{j}, j \neq i, j=1, \cdots, \mathscr{N}$.

\section{Partial eigenstructure assignment problem}

Assigning $m$ of the closed-loop eigenvalues to a certain negative value can be performed through the LMI characterization presented in the previous section. The problem is to partially assign the set of eigenvalues

$$
\{\overbrace{\delta, \cdots, \delta}^{m \text { times }}\}
$$

of $A_{0}+B_{2} F$ by state feedback, which can be dealt with in two steps:

1) compute the base $\left[\begin{array}{l}M_{\delta} \\ N_{\delta}\end{array}\right]$ of nullspace of $\left[\begin{array}{lll}A_{0}-\delta I & B_{2}\end{array}\right]$ with conformable partitioning;

2) with arbitrary $\varepsilon_{k} \in \mathbb{R}^{m}, k=1, \cdots, m$, the state feedback will be obtained as $K=Y G^{-1}$ with

$$
Y=N \Gamma_{N}, G=M \Gamma_{M},
$$

in which

$$
\begin{aligned}
N & :=[\overbrace{N_{\delta}, \cdots, N_{\delta}}^{\text {m times }}, \overbrace{I, \cdots, I}^{(n-m) \text { times }}], \\
M & :=[\overbrace{M_{\delta}, \cdots, M_{\delta}}^{\text {m times }}, \overbrace{I, \cdots, I}^{(n-m) \text { times }}], \\
\Gamma_{N} & :=\operatorname{diag}\left(\varepsilon_{1}, \cdots, \varepsilon_{m}, \lambda_{1}, \cdots, \lambda_{(n-m)}\right), \\
\Gamma_{M} & :=\operatorname{diag}\left(\varepsilon_{1}, \cdots, \varepsilon_{m}, \beta_{1}, \cdots, \beta_{(n-m)}\right)
\end{aligned}
$$

with $\lambda_{k} \in \mathbb{R}^{n}$ and $\beta_{k} \in \mathbb{R}^{n}$. Note that only vectors $\eta_{k}$ are related to the assignment of the $m$ eigenvalues to $\delta$. In other words, other vectors $\left(\lambda_{k}\right.$ and $\left.\beta_{k}\right)$ are not exploited in the pole placement purposes and thereby can be employed to meet other Lyapunov-type constraints.

Now, provided by the LMI characterization in (11), (12) and (13), the first step of our multi-channel $\mathscr{H}_{2}$-based SMC design can be set as an LMI program in the variables $X_{i}>0$, $Z_{i}>0, i=1, \cdots, \mathscr{N}, \Gamma_{M}, \Gamma_{N}$ and $\gamma_{i}>0$, by recasting (14) as:

minimize $\gamma_{i}$

subject to (11), (12), (13), and (16) for i-th channel, (11), 12), (13), and (16) for $\mathrm{j}$-th channel with given $\gamma_{j}, j \neq i, j=1, \cdots, \mathscr{N}$.

However, we have not yet shown that the set of closedloop eigenvalues encompasses (15). This is the subject of the following lemma.

Lemma 2: The set of the eigenvalues of $A_{0}+B_{2} K$, obtained by letting the state feedback $K=Y G^{-1}$, with $Y$ and $G$ given in (16), contains the subset (15).

Proof: From (16), we can write

$$
\begin{aligned}
& \left(A_{0}+B_{2} K-\delta I\right) M_{\delta} \varepsilon_{k} \\
= & {\left[A_{0}+B_{2}\left(N \Gamma_{N}\right)\left(M \Gamma_{M}\right)^{-1}-\delta I\right] M_{\delta} \varepsilon_{k} } \\
= & {\left[A_{0}+B_{2}\left(N \Gamma_{N}\right)\left(M \Gamma_{M}\right)^{-1}-\delta I\right]\left(M \Gamma_{M}\right) e_{k} } \\
= & {\left[A_{0}\left(M \Gamma_{M}\right)+B_{2}\left(N \Gamma_{N}\right)-\delta\left(M \Gamma_{M}\right)\right] e_{k} } \\
= & A_{0} M_{\delta} \varepsilon_{k}+B_{2} N_{\delta} \varepsilon_{k}-\delta M_{\delta} \varepsilon_{k} \\
= & \delta M_{\delta} \varepsilon_{k}-\delta M_{\delta} \varepsilon_{k} \\
= & 0, \quad k=1, \cdots, m .
\end{aligned}
$$

Note that $e_{k}$ denotes the canonical basis of $\mathbb{R}^{n}$. The above manipulation shows that $\delta$ is a solution of the characteristic equation $\operatorname{det}\left(s I-A_{0}-B_{2} K\right)=0$, repeated $m$-times.

Remark 2: Rather than the proposed partial eigenstructure assignment in the first stage, it is also possible to exploit a pure pole placement method. This means that the poles that govern the sliding motion are known and will be 
assigned during the designing procedure. However, this can limit the degrees of freedom in the problem, especially for single input systems, so that no more freedom remains for other performance constraints. Notice that this eigenstructure assignment scheme is significantly different from the ones explained in [9], [10], [11], in which the control effort, required to induce and maintain sliding, is not taken into account.

\section{Stability of The Reduced-Order Dynamics AND ObTAINING The SLIDING MATRIX}

This section proposes an approach to obtain the sliding matrix $S$ related to the matrix gain $K$, derived based on the partial eigenstructure assignment scheme in the previous section, while ensuring the stability of the resulting reduced order dynamics. The approach is built based on the regular form scheme. Consider a change of coordinates $x \mapsto T_{r} x$. In this new coordinate system, the system matrices are of the form:

$$
\tilde{A}_{i}=\left[\begin{array}{ll}
A_{i 11} & A_{i 12} \\
A_{i 21} & A_{i 22}
\end{array}\right], \tilde{B}_{2}=\left[\begin{array}{c}
0 \\
B_{p}
\end{array}\right]
$$

where the square matrix $B_{p} \in \mathbb{R}^{m \times m}$ has full rank and more importantly is nonsingular; see [12]. Now, the switching function matrix in the original coordinates is parameterized such that [12]

$$
F=F_{2}\left[\begin{array}{ll}
-\mathscr{M} & I_{m}
\end{array}\right] T_{r},
$$

where $F_{2} \in \mathbb{R}^{m \times m}$ and $\mathscr{M}=-F_{2}^{-1} F_{1} \in \mathbb{R}^{m \times(n-m)}$ is an unknown matrix which will be derived hereafter. Notice that theoretically the choice of $F_{2}$ may not influence the sliding motion [12]. Let $\left(\tilde{x}_{1}, \tilde{x}_{2}\right)$ be the partition of the system states associated with the certain system coordinates in (19), then it can readily be shown that while the system states are confined to the sliding manifold, ie, $s=0$, the reducedorder sliding mode dynamics are governed by the following reduced order system

$$
\dot{\tilde{x}}_{1}(t)=\sum_{i=0}^{h}\left(A_{i 11}+A_{i 12} \mathscr{M}\right) \tilde{x}_{1}\left(t-\tau_{i}\right) .
$$

As a result, the matrix $\mathscr{M}$ can be considered as a statefeedback matrix that stabilizes the reduced order dynamics. Consider the following LyapunovKrasovskii functional:

$$
\tilde{V}(t)=\tilde{x}_{1}^{T}(t) \tilde{P}_{0} \tilde{x}_{1}(t)+\sum_{i=1}^{h} \int_{t-\tau_{i}}^{t} \tilde{x}_{1}^{T}\left(\beta-\tau_{i}\right) \tilde{P}_{i} \tilde{x}_{1}\left(\beta-\tau_{i}\right) d \beta,
$$

where $\tilde{P}_{i}>0, i=1, \cdots, h$. Now, in order to ensure the asymptotic stability of the reduced-order dynamics, we let $\dot{\bar{V}}(t)<0$. It follows then

$$
\begin{aligned}
\dot{\tilde{V}}(t)=2 \tilde{x}_{1}^{T}(t) \tilde{P}_{0} \dot{\tilde{x}}_{1}(t) & +\sum_{i=1}^{h} \tilde{x}_{1}^{T}(t) \tilde{P}_{i} \tilde{x}_{1}(t) \\
& -\sum_{i=1}^{h} \tilde{x}_{1}^{T}\left(t-\tau_{i}\right) \tilde{P}_{i} \tilde{x}_{1}\left(t-\tau_{i}\right)<0 .
\end{aligned}
$$

Using the equation (21) in (23), it can be obtained

$$
\left[\begin{array}{cc}
\operatorname{herm}\left(\tilde{P}_{0}\left(A_{011}+A_{012} \mathscr{M}\right)\right)+\sum_{i=1}^{h} \tilde{P}_{i} & \star \\
\operatorname{vec}\left(\left(A_{i 11}+A_{i 12} \mathscr{M}\right)^{T} \tilde{P}_{0}\right)_{i=1}^{h} & -\operatorname{diag}\left(\tilde{P}_{i}\right)_{i=1}^{h}
\end{array}\right]<0,
$$

or by introducing $P_{i}:=\tilde{P}_{i}^{-1}$ and $Q_{0}:=\mathscr{M} P_{0}$ and using the Schur complement:

$$
\left[\begin{array}{cc}
\operatorname{herm}\left(A_{011} P_{0}+A_{012} Q_{0}\right)+\sum_{i=1}^{h} H_{i} & \star \\
\operatorname{vec}\left(\left(A_{i 11} P_{0}+A_{i 12} Q_{0}\right)^{T}\right)_{i=1}^{h} & -\operatorname{diag}\left(H_{i}\right)_{i=1}^{h}
\end{array}\right]<0,
$$

where $H_{i}:=P_{0}^{-1} P_{i} P_{0}^{-1}$.

Suppose that $\tilde{K}$ denotes the state feedback, derived based on the partial eigenstructure assignment scheme, in the new coordinate. Then, to obtain the associated sliding matrix, we equate $\tilde{K}$ and the linear controller in (6) in the new coordinate. Assume that $\delta$ does not belong to the spectrum of $\tilde{A}_{0}$, ie, $\left(\tilde{A}_{0}-\delta I_{n}\right)$ is invertible, and let

$$
\tilde{K}\left(\tilde{A}_{0}-\delta I_{n}\right)^{-1}=\left[\begin{array}{ll}
\mathscr{K}_{1} & \mathscr{K}_{2}
\end{array}\right]
$$

where $\mathscr{K}_{1} \in \mathbb{R}^{m \times(n-m)}$. Then, we can obtain

$$
\mathscr{M}=B_{p} \mathscr{K}_{1} \text {. }
$$

Further, since $\mathscr{M}=Q_{0} P_{0}^{-1}$ from 25:

$$
Q_{0} P_{0}^{-1}=B_{p} \mathscr{K}_{1},
$$

or by right multiplication of both sides of the above equality to $P_{0}$

$$
Q_{0}=B_{p} \mathscr{K}_{1} P_{0} .
$$

The condition in 29] can be dealt with a simple relaxation method as:

$$
\begin{aligned}
& \text { minimize } \alpha \text { subject to } \\
& \text { (25) and }\left\|Q_{0}-B_{p} \mathscr{K}_{1} P_{0}\right\|<\alpha,
\end{aligned}
$$

where $\alpha>0$ is a scalar variable. Simply it can be shown that the above problem is equivalent to the following LMI minimization problem:

$$
\begin{aligned}
& \text { minimize } \alpha \text { subject to }[25 \text { and } \\
& {\left[\begin{array}{cc}
-\alpha I & \star \\
Q_{0}-B_{p} \mathscr{K}_{1} P_{0} & -\alpha I
\end{array}\right]<0 .}
\end{aligned}
$$

Hence, the multi-channel $\mathscr{H}_{2}$ based SMC problem is to find the global solution of the above minimization problem and then the switching function matrix $F$ can be derived using (20). In the case of feasibility, the above optimization problem will enforce $\alpha$ to be an extremely small number associated with the precision of the computational unit. Now, the switching function matrix $F$ can be derived using (20).

Remark 3: Note that as the spectrum of the closedloop matrix $\tilde{A}_{0}+\tilde{B}_{2} \tilde{K}$ includes $m$ repeated eigenvalues $\delta$, the spectrum of $\tilde{A}_{0}-\delta I_{n}+\tilde{B}_{2} \tilde{K}$ includes $m$ repeated zero eigenvalue. Hence, it can be shown that there always exists a matrix $\tilde{F}$ such that $\tilde{F}\left(\tilde{A}_{0}-\delta I_{n}+\tilde{B}_{2} \tilde{K}\right)=0$. This statement is equivalent to the equality 27, employed for obtaining the switching matrix. 


\section{Finite Time Reachability Analysis of The Designed Optimal SLiding SuRface}

This section aims to find a condition under which the controller (5), (4) drives the system state to the sliding surface (2) in finite time.

Proposition 1: If $\left\|\sum_{i=1}^{h} A_{i} x\left(t-\tau_{i}\right)\right\| \leq \psi$, with $\psi>0$ a constant scalar, the states of the closed-loop system, obtained by applying the controller (5), (4) to the system in (1), reach to the sliding surface (2) and remain there thereafter if the gain $\varphi>0$ satisfies

$$
\varphi>\|F\| \psi,
$$

where $F$ is a given sliding function matrix.

Proof: Let us take the time derivative of (2), substitute $\dot{x}$ as the state equation (1), and use the controller (5), (4), we may obtain then

$$
\begin{aligned}
\dot{s}(t)= & \delta s(t)+F \sum_{i=1}^{h} A_{i} x\left(t-\tau_{i}\right)-\left[\varphi+\left\|F B_{2}\right\| \rho(x, u, t)\right] \frac{s(t)}{\|s(t)\|} \\
& +F B_{2} f(x, u, t) .
\end{aligned}
$$

Now, it is required to show that the following reachability condition is satisfied:

$$
\frac{s^{T} \dot{s}}{\|s\|}<0 .
$$

It follows from (33) and $\left\|\sum_{i=1}^{h} A_{i} x\left(t-\tau_{i}\right)\right\| \leq \psi$ that

$$
\begin{aligned}
\frac{s^{T} \dot{s}}{\|s\|} & \leq \delta\|s\|+\|F\| \psi-\varphi+\left\|F B_{2} f(x, u, t)\right\|-\left\|F B_{2}\right\| \rho(x, u, t) \\
& \leq\|F\| \psi-\varphi .
\end{aligned}
$$

Finally, if $\varphi$ satisfies (32), the reachability condition (34) holds.

\section{NUMERICAL EXAMPLE}

Consider a second order single delay system in form of (1) with the following data:

$$
A_{0}=\left[\begin{array}{ll}
0 & 1 \\
0 & 0
\end{array}\right], A_{1}=\left[\begin{array}{ll}
1 & 0 \\
0 & 0
\end{array}\right], B_{2}=\left[\begin{array}{l}
0 \\
1
\end{array}\right], \tau=1 s .
$$

The above system is considered in [13], and with no time delay, ie, $A_{1}=0$, is the first example of [3]. As stated in [3], if $A_{1}=0$, the closed-loop system obtained by applying the state feedback obtained from the standard LQR problem, with any given $C_{2}$ and $D_{2}=\varsigma$, for all choices of $\varsigma>0$, is governed by a complex poles with a damping ratio of $\zeta=0.7071$ [3], [14]. This feedback gain cannot be assumed as the linear part of a sliding mode controller. Now let $C=[105], D=0.24$, $C_{2}=\left[\begin{array}{ll}10 & 0\end{array}\right]$ and $D_{2}=1$.

We solve (18), with $B_{1}=I$, by letting $\gamma_{2}=150, \delta=10$, the state feedback gain is obtained as

$$
K=\left[\begin{array}{ll}
-16.9756 & -11.6976
\end{array}\right],
$$

By employing the given approach, the switching function matrix is

$$
F=\left[\begin{array}{ll}
1.6976 & 1.0000
\end{array}\right]
$$

(a)

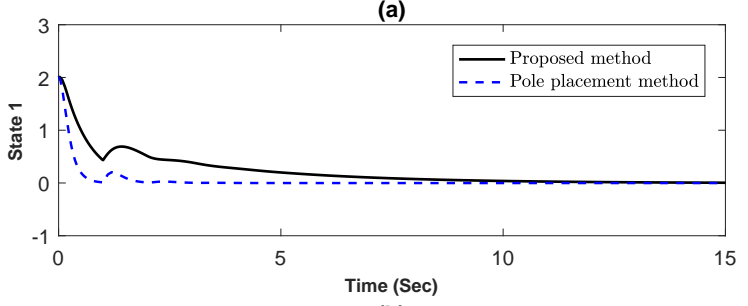

(b)

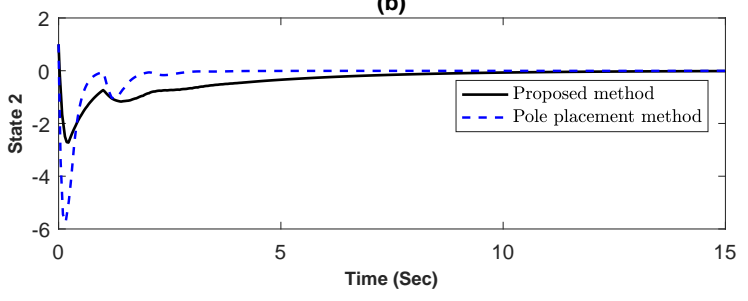

Fig. 1. Evolution of the closed-loop system state trajectories

The poles of $A_{0}+B K$ are at $\{-10,-1.6976\}$, and the upper bound of $\mathscr{H}_{2}$ cost from $w$ to $z_{1}$ and $z_{2}$, when the system in (1) is without time delay, are 3.5669 and 7.6074, respectively. For comparison, we also find the switching function matrix by placing the poles of $A_{0}+B K$ at $\{-10,-5.9000\}$. In such a case, the switching function matrix is

$$
F=\left[\begin{array}{ll}
5.9000 & 1.0000
\end{array}\right] .
$$

In this case, the upper bound of $\mathscr{H}_{2}$ cost from $w$ to $z_{1}$ and $z_{2}$, when the system in (1) is without time delay, are 6.5167 and 11.6595 , respectively. We further let the matched uncertainty term in (1) be $f(x, u, t)=x_{1}^{2}(t)+0.1 \sin (t) x_{2}(t)$. The discontinuity in the nonlinear control term $\xi(t)$ in (4) is also smoothed by using a sigmoidal approximation [15] as

$$
\xi_{\varepsilon}(t)=-\left(F B_{2}\right)^{-1}\left[\varphi+\left\|F B_{2}\right\| \rho(x, u, t)\right] \frac{s(t)}{\varepsilon+\|s(t)\|}
$$

with the scalar $\varepsilon=0.05$ which this can remove the discontinuity at $s=0$ and introduce the possibility to accommodate the actuator rate limits. We further let $\psi=2$ $(\varphi=2\|F\|+0.01)$ and $\rho(x, u, t)=4.3$ in (36). Fig. 1 shows the trajectories of the system state by the initial condition of $x(0)=\left[\begin{array}{ll}2 & 1\end{array}\right]^{T}$, and using the switching surfaces and state feedback gains obtained above. The corresponding control signals and switching function are also demonstrated in Fig. 2. As seen, the proposed method here requires less control efforts, in comparison with the control law obtained as the solution of the pole placement problem explained above, for stabilizing the system.

\section{CONCLUSIONS}

This paper has been devoted to the development of a novel method for the design of an SMC, whose switching surface is derived from an optimization problem constructed to meet a number of Lyapunov-type performance constraints, for multiple time delay systems. In doing so, in the first stage, through a convex optimization approach, a state feedback gain is found while assigning a certain number $(m)$ of the closedloop system eigenvalues to a predetermined negative value, 


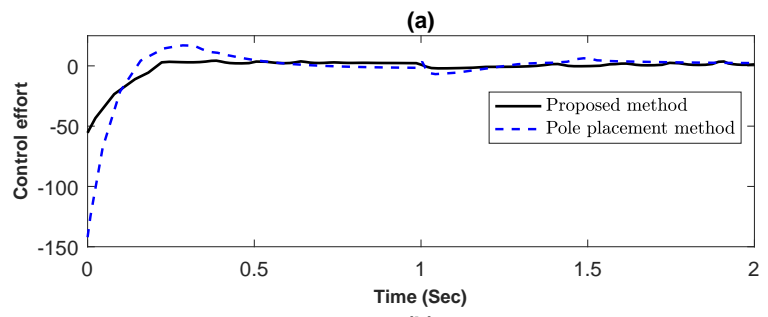

(b)

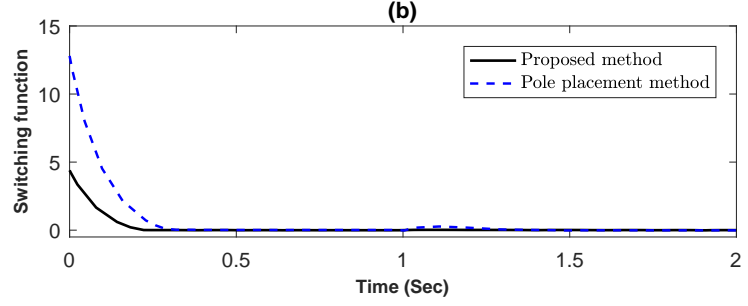

Fig. 2. (a) Control efforts (b) Evolution of the switching function

as well as satisfying multi-channel $\mathscr{H}_{2}$-norm constraints. Then, the proposed second stage finds the associated sliding surface while ensuring the stability of the associated reduced order dynamics. The advantages of the proposed scheme are threefold: (a) it can set the stage for designing SMC while the level of control efforts is taken into account; (b) it makes it possible to integrate a number of Lyapunov-type constraints, e.g. regional pole placement constraints, into the SMC design problem; (c) the controller can be computed in a numerically very efficient method. The achieved results, in the simulation section, confirmed the effectiveness of the developed scheme.

\section{REFERENCES}

[1] X. Han, E. Fridman, and S. K. Spurgeon, "Sliding-mode control of uncertain systems in the presence of unmatched disturbances with applications," International Journal of Control, vol. 83, no. 12, pp. 2413-2426, 2010.

[2] S. Xu, J. Lam, and Y. Zou, "New results on delay-dependent robust $H_{\infty}$ control for systems with time-varying delays," Automatica, vol. 42, no. 2 , pp. $343-348,2006$.

[3] C. Edwards, "A practical method for the design of sliding mode controllers using linear matrix inequalities," Automatica, vol. 40, pp. 1761-1769, 2004.

[4] Y. Pan and K. Furuta, "VSS controller design for discrete-time systems," Control-Theory Adv. Technol, vol. 10, no. 4, pp. 669-687, 1994.

[5] C. Y. Tang and E. A. Misawab, "Sliding surface design for discrete VSS using LQR technique with a preset real eigenvalue," Systems and Control Letters, vol. 45, no. 1, pp. 1-7, Jan, 2002.

[6] M. C. De Oliveira and J. C. Geromel, "Synthesis of non-rational controllers for linear delay systems," Automatica, vol. 40, no. 2, pp. 171-188, 2004.

[7] M. De Oliveira, J. Gerome, and J. Bernussou, "An LMI optimization approach to multiobjective controller design for discrete-time systems," in Decision and Control, 1999. Proceedings of the 38th IEEE Conference on, vol. 4. IEEE, 1999, pp. 3611-3616.

[8] C. Scherer, P. Gahinet, and M. Chilali, "Multiobjective outputfeedback control via LMI optimization," IEEE Transactions on Automatic Control, vol. 42, no. 7, pp. 896-911, 1997.

[9] C. Edwards and S. K. Spurgeon, Sliding Mode Control: Theory and Applications. London: Taylor and Francis, 1998.

[10] C. Dorling and A. Zinober, "Two approaches to hyperplane design in multivariable variable structure control systems," International Journal of Control, vol. 44, no. 1, pp. 65-82, 1986.

[11] — "Robust hyperplane design in multivariable variable structure control systems," International Journal of Control, vol. 48, no. 5, pp. 2043-2054, 1988.
[12] V. I. Utkin, Sliding Modes in Control Optimization, Communications and Control Engineering Series. London: Springer-Verlag, 1992.

[13] A. E. Pearson and Y. A. Fiagbedzi, "An observer for time lag systems," IEEE Transactions on Automatic Control, vol. 34, no. 7, pp. 775-777, 1989.

[14] G. Franklin, J. Powell, and E. A. Naeini, Feedback Control of Dynamic Systems. Prentice Hall, 2002.

[15] H. Alwi and C. Edwards, "Fault tolerant control using sliding modes with on-line control allocation," in Fault Tolerant Flight Control. Springer, 2010, pp. 247-272.

\section{APPENDIX}

\section{A. Proof of Lemma 1}

The equivalence between the first two statements can be proved using the method employed e.g. in [6, Lemma 2]. We just show the equivalence between the statements $i i)$ and iii). Let

$$
\Xi=\left[\begin{array}{cccc}
X & \star & \star & \star \\
0 & \Phi_{22} & \star & \star \\
0 & 0 & -v^{-1} \operatorname{diag}\left(W_{i}\right)_{i=1}^{h} & \star \\
0 & 0 & 0 & -v^{-1} \gamma I
\end{array}\right]
$$

With well-known Schur complement, it can readily be shown that the first LMI in iii) can be reformulated as

$$
\Xi+\operatorname{herm}\left(\left[\begin{array}{c}
-I \\
I+v\left(A_{0}+B_{2} K\right) \\
\operatorname{vec}(I)_{i=1}^{h} \\
C+D K
\end{array}\right] G\left[\begin{array}{c}
I \\
0 \\
\operatorname{vec}(\mathbf{0})_{i=1}^{h} \\
0
\end{array}\right]^{T}\right)<0 .
$$

Using the well-known Projection lemma, it can be readily shown that 38, holds iff

$$
\Omega_{1}^{T} \Xi \Omega_{1}<0,
$$

and

$$
\Omega_{2}^{T} \Xi \Omega_{2}<0
$$

where

$$
\begin{aligned}
& \Omega_{1}=\left[\begin{array}{ccc}
I+v\left(A_{0}+B_{2} K\right)^{T} & \sqrt{v}\left(\operatorname{vec}(I)_{i=1}^{h}\right)^{T} & \sqrt{v}(C+D K)^{T} \\
I & 0 & 0 \\
0 & \operatorname{diag}(\sqrt{v} I)_{i=1}^{h} & 0 \\
0 & 0 & \sqrt{v} I
\end{array}\right], \\
& \Omega_{2}=\left[\begin{array}{ccc}
0 & 0 & 0 \\
I & 0 & 0 \\
0 & \sqrt{v} \operatorname{diag}(I)_{i=1}^{h} & 0 \\
0 & 0 & \sqrt{v} I
\end{array}\right] .
\end{aligned}
$$

Notice that $X>0$. It can be seen that (40) is implied by (39). Further, 39) can be rewritten as

$$
\begin{aligned}
& {\left[\begin{array}{ccc}
\left(\text { herm }\left[v\left(A_{0}+B_{2} K\right) X\right]+v \sum_{i=1}^{h} A_{i} W_{i} A_{i}^{T}\right. & \star & \\
\sqrt{v} \operatorname{vec}(X)_{i=1}^{h} & -\operatorname{diag}\left(W_{i}\right)_{i=1}^{h} & \star \\
\sqrt{v}(C+D K) X & 0 & -\gamma I
\end{array}\right]} \\
& +\left[\begin{array}{c}
v\left(A_{0}+B_{2} K\right) \\
\sqrt{\nu} \operatorname{vec}(I)_{i=1}^{h} \\
\sqrt{v}(C+D K)
\end{array}\right] X\left[\begin{array}{c}
v\left(A_{0}+B_{2} K\right) \\
\sqrt{\nu} \operatorname{vec}(I)_{i=1}^{h} \\
\sqrt{v}(C+D K)
\end{array}\right]^{T} .
\end{aligned}
$$

By performing congruence transformation $\left[\frac{1}{\sqrt{v}} I\left(\operatorname{vec}(I)_{i=1}^{h}\right)^{T} I\right]$ in the above inequality and introducing $Y=K X$, it can be readily seen that the feasibility of the obtained inequality is equivalent to that of the first LMI in item $i i)$. 